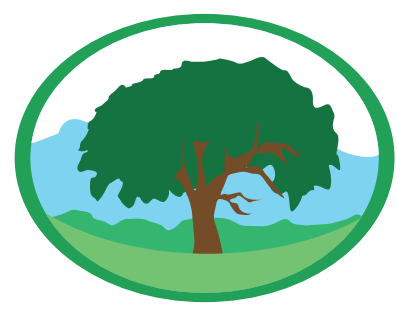

\title{
Habitat de cinco espécies de peixes nativos do rio Formoso, Minas Gerais
}

GUEDES, H.A.S. ${ }^{1}$, SILVA, D.D. ${ }^{2}$, DERGAM, J.A. ${ }^{3}$, MARTINS, V.S. ${ }^{4}$

${ }^{1}$ Eng ${ }^{\circ}$. Civil, Prof. Adjunto, Centro de Engenharias, UFPel/Pelotas - RS, Fone: (53) 3225-4573, hugo.guedesQufpel.edu.br.

${ }^{2}$ Agrônomo, Prof. Titular, Departamento de Engenharia Agrícola, UFV/Viçosa - MG, demetrius.ufvagmail.com.

${ }^{3}$ Biólogo, Prof. Titular, Departamento de Biologia Animal, UFV/Viçosa - MG, jdergamagmail.com.

${ }^{4}$ Eng ${ }^{\circ}$. Agrícola e Ambiental, Mestrando no Programa de Pós-Graduação em Sensoriamento Remoto, INPE/São Paulo - SP, vitormartins9ahotmail.com.

\section{Palavras-chave:}

Biodiversidade. Índice de Uso de Habitat. Rios tropicais.

Vazões ecológicas.

\begin{abstract}
Resumo
0 objetivo deste estudo foi descrever a relação entre o uso do habitat e as variáveis velocidade, profundidade e substrato, para cinco espécies de peixes nativos, em dois trechos no rio Formoso, Minas Gerais. Foram realizadas quatro coletas de dados em dois trechos de rio, sendo identificados como dominantes dois Characiformes (Astyanax bimaculatus e Leporinus steindachneri), dois Siluriformes (Trachelyopterus striatulus e Hypostomus affinis) e um Perciformes (Geophagus brasiliensis). Os usos dos habitats para cada espécie dominante foram caracterizados utilizando-se as variáveis velocidade da água, profundidade do escoamento e tipo de substrato. As espécies de menor porte apresentaram exemplares mais presentes no trecho 2 , de escoamento lento e mais degradado. Já as espécies de médio a grande porte apresentaram mais indivíduos no trecho 1 , de escoamento rápido e mais preservado. Os índices de uso de habitat e as curvas de frequência obtidos neste estudo são fundamentais para subsidiar os estudos de modelagem e determinação de vazões ecológicas no rio Formoso, sendo ferramenta útil na tomada de decisão de órgãos ambientais.
\end{abstract}

\section{Habitat of five native fish species of the Formoso river, Minas Gerais}

Key words: Biodiversity. Environmental flow. Habitat Suitability Index. Tropical rivers.

\begin{abstract}
The study aimed to describe the relationship between the habitat use and the velocity, depth and substrate variables of five native fish species of the Formoso River, Minas Gerais. Four data collection at two stretches of the river were done, being identified like dominant two Characiformes (Astyanax bimaculatus and Leporinus steindachneri), two Siluriformes (Trachelyopterus striatulus and Hypostomus affinis) and one Perciformes (Geophagus brasiliensis). The habitats uses for each dominant species were characterized using the variables water velocity, flow depth and substrate type. The smaller species have submitted more individuals at stretch 2, characterized by presenting a slow flow regime and most degraded river. The larger species have submitted more individuals at stretch 1 , characterized by presenting a fast flow regime and less degraded river. The habitat use indices and the frequency curves obtained in this study are fundamental to support the modeling studies and determination of environmental flows in the Formoso River, and useful tool in the decision making of environmental agencies.
\end{abstract}




\section{INTRODUÇÃO}

O estudo do habitat é uma etapa fundamental para a compreensão dos padróes de distribuição de peixes em diferentes mananciais (COSTA et al., 2013). Entretanto, grande parte das pesquisas que tratam sobre esse assunto é baseada em usos de habitat de peixes nativos de águas doces temperadas (SOUZA et al., 2013). No Brasil, poucos esforços têm sido feitos para compreender as diferenças de uso de habitat de peixes de águas doces neotropicais (TERESA; CASATTI, 2013).

A investigação das assembleias de peixes e o estudo do uso de habitat podem servir para melhorar a capacidade avaliativa das consequências biológicas de impactos antrópicos sobre os ecossistemas aquáticos, auxiliando gestores ambientais a estabelecer políticas públicas para a conservação da biodiversidade em escalas local e regional (BARROCA et al., 2012).

A distribuição de peixes por diferentes habitats é frequentemente utilizada na modelagem ecohidrológica (MARSILI-LIBELLI et al., 2013; GUEDES et al., 2014; CASTRO et al., 2016) para avaliar a necessidade de vazóes ecológicas, geralmente baseada na metodologia denominada Instream Flow Incremental Methodology (IFIM) (BOVEE, 1982). A metodologia IFIM baseia-se no princípio de que a distribuição longitudinal e lateral dos organismos aquáticos é determinada, entre outros fatores, pelas características hidráulicas, estruturais e morfológicas dos cursos d'água.

O IFIM permite que os gestores de recursos hídricos quantifiquem o regime de variaçáo de vazóes, natural ou modificado, para as espécies selecionadas como indicadoras, sendo que, em condiçôes adequadas, é o melhor método para estabelecer uma vazão mínima para essas espécies (STALNAKER et al., 1995).

A heterogeneidade dos habitats em ambientes lóticos geralmente influencia a distribuição de peixes, potencializando à medida que aumenta a complexidade do habitat dos cursos d'água (TERRA et al., 2010). Diversas variáveis ambientais são preferencialmente selecionadas por espécies de peixes nesses ecossistemas (DEVINE; MUNDAY, 2013), sendo que grande parte dos modelos de uso de habitat utiliza as variáveis velocidade da água, profundidade do escoamento (KAUFMANN; PINHEIRO, 2009) e tipo de substrato (COSTA et al., 2013).

O rio Formoso, localizado no estado de Minas Gerais, é um importante afluente do rio Pomba, o qual por sua vez é afluente da bacia do rio Paraíba do Sul. A extensa bacia do Paraíba do Sul é considerada estratégia na região Sudeste por incluir drenagens nos estados de São Paulo, Minas Gerais e Rio de Janeiro. Atualmente, o rio Formoso possui características distintas, sendo que da porção média à cabeceira predominam áreas mais preservadas com a presença de mata ciliar e condiçôes naturais. Já próximo ao seu encontro com o rio Pomba, a região está degradada e a vegetação nativa deu lugar a pastagens, com ausência de mata ciliar e avançado processo de assoreamento das margens e degradação da qualidade da água, além de sofrer forte influência da urbanizaçáo. Pelo fato de ter regióes preservadas e degradadas, por ser um importante afluente na região e por não apresentar uma descrição do uso de habitat para diferentes espécies de peixes, o rio Formoso apresenta condiçóes adequadas para o estudo pretendido.

Sendo assim, o objetivo deste estudo foi descrever a relaçâo entre o uso do habitat e as variáveis velocidade, profundidade e substrato, para cinco espécies de peixes nativos, em dois trechos no rio Formoso em Minas Gerais. O estudo foi realizado com o intuito de verificar a hipótese de que a distribuição dos indivíduos coletados difere em função das diferenças locais e utilizam trechos do rio com diferentes características hidráulicas e tipos de substratos.

\section{MATERIAL E MÉTODOS}

$\mathrm{O}$ rio Formoso, afluente da margem direita do rio Pomba, possui $76,7 \mathrm{~km}$ de extensão e localiza-se na região oeste da bacia hidrográfica do rio Paraíba do Sul entre os paralelos $21^{\circ} 18^{\prime}$ e $21^{\circ} 27^{\prime}$ Sul e os meridianos $43^{\circ} 10^{\prime}$ e $43^{\circ} 38^{\prime}$ Oeste (Figura 1; Tabela 1). 
Tabela 1. Características gerais dos dois trechos estudados no rio Formoso

\begin{tabular}{llllll} 
Trechos & $\begin{array}{l}\text { Regime de } \\
\text { escoamento } \\
\text { hidráulico }\end{array}$ & $\begin{array}{l}\text { Faixa de largura do } \\
\text { rio }(\mathrm{m})\end{array}$ & $\begin{array}{l}\text { Faixa de } \\
\text { profundidade }(\mathrm{m})\end{array}$ & Faixa de altitude (m) & Vazão média (m3 s-1) \\
\hline 1 & Torrencial & $12,0-14,0$ & $0,2-1,4$ & $460-473$ & 4,9 \\
2 & Fluvial & $7,0-8,0$ & $1,0-2,0$ & $427-429$ & 7,4
\end{tabular}

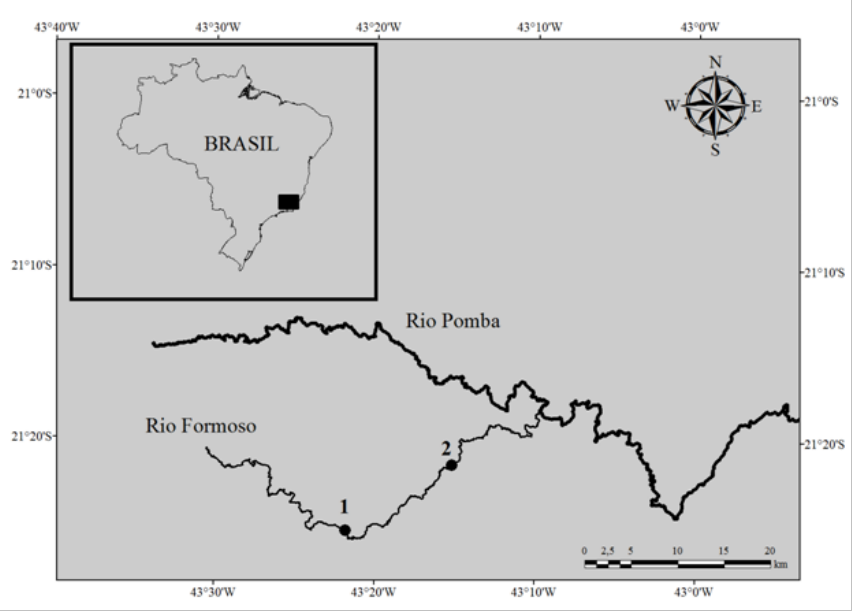

Figura 1. Localização geográfica da área de estudo, indicando o início dos dois trechos de estudo

Analisou-se a relação entre o micro-habitat e os usos dos peixes dominantes em dois trechos do rio Formoso, distantes em aproximadamente $14 \mathrm{~km}$. O trecho $1\left(21^{\circ} 25^{\prime} 23.5^{\prime \prime} \mathrm{S}, 43^{\circ} 21^{\prime} 45.0^{\prime \prime W}\right)$, com área de contribuição de $180,7 \mathrm{~km}^{2}$, localiza-se na porção média

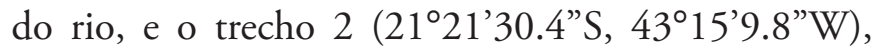
com área de contribuição de $312,3 \mathrm{~km}^{2}$, localiza-se próximo à foz do rio.

As amostragens de peixes, variáveis hidráulicas e substratos foram quantificados in situ em quatro coletas no rio Formoso: março e junho de 2011, fevereiro e julho de 2012. As coletas foram conduzidas em dois trechos de um km de extensão, onde se demarcou três seçôes transversais equidistantes de 500 metros, nas quais foram determinados os valores de velocidade da água, profundidade do escoamento, vazão; identificados diferentes tipos de sedimentos no fundo do rio (substrato) e as espécies de peixes dominantes na área de estudo. A definição das espécies dominantes no rio Formoso seguiu a metodologia apresentada por Guedes et al. (2014).

A velocidade do escoamento foi monitorada durante as duas primeiras campanhas de campo utilizando-se um micromolinete hidráulico M1 da SEBA Hydrometrie' nas seçôes transversais demarcadas. $\mathrm{Na}$ terceira e quarta campanha de campo, a velocidade foi monitorada por meio de um molinete fluviométrico Newton da Hidromec®. A profundidade do escoamento foi medida por meio de levantamentos batimétricos das seçôes transversais no mesmo momento em que eram efetuadas as mediçóes de velocidade da água.

A coleta de substratos no fundo do canal, totalizando 30 amostras por seção, foi realizada por meio de uma draga tipo Petersen da Hidromec $₫$ de penetração vertical e capacidade de coleta de 3,20 litros. Os materiais coletados foram encaminhados para o Laboratório de Propriedades Físicas do Solo, do Departamento de Solos da Universidade Federal de Viçosa (UFV), para análise da granulometria dos agregados. Os substratos foram assim classificados: argila $(<0,004 \mathrm{~mm})$; silte $(0,004$ a $0,06 \mathrm{~mm})$; areia $(0,06$ a 2,0 mm); cascalho (2,0 a 64,0 mm); pedra (> $64,0 \mathrm{~mm})$.

As amostragens de peixes foram realizadas nas mesmas seções onde foram determinados o substrato, a velocidade e a profundidade do escoamento, totalizando 24 amostras (4 coletas de dados $\mathrm{x} 2$ trechos x 3 seçôes). Em cada seção de monitoramento foram posicionadas redes de espera no período da tarde e retiradas na manhã do dia seguinte, de modo a ficarem por um período médio de 12 horas. Foram dispostas em cada seção de monitoramento sete redes de espera com malhas de 0,$015 ; 0,020 ; 0,025 ; 0,030 ; 0,035$; 0,040 e $0,050 \mathrm{~m}$, equidistantes de 10 metros a jusante da seção transversal monitorada. As sete redes foram armadas exatamente onde as variáveis velocidade, profundidade e substrato foram monitoradas.

No final das coletas, os exemplares de peixes foram conduzidos ao Laboratório de Sistemática Molecular Beagle, do Departamento de Biologia Animal UFV, para dar sequência à identificação das espécies. A identificação das espécies foi feita com base em literatura específica de cada grupo (BRITSKI et al., 1986; MENEZES, 1987; REIS et al., 2003). 
O uso do habitat foi caracterizado pela frequência com que uma determinada espécie foi observada, em função das variáveis profundidade, velocidade e substrato. Sendo assim, as espécies dominantes foram separadas em classes de profundidade e velocidade, e códigos de substratos, de acordo com a metodologia proposta por Costa et al. (2013). A porcentagem de ocorrência de peixes em classes de frequência de profundidade, velocidade e substratos foi determinada relacionando a quantidade de indivíduos em cada classe com o somatório dos indivíduos nos dois trechos de estudo (Equação 1).

$$
P_{i}=\left(\frac{N_{i}}{\sum N_{i}} x 100 ; \quad i=1 \ldots n\right)
$$

em que, $\mathrm{P}_{\mathrm{i}}=$ porcentagem de ocorrência de peixes separadas em classes de habitat $\mathrm{i} ; \mathrm{N}_{\mathrm{i}}=$ número de indivíduos em classes de habitat i de todos os trechos, e $\mathrm{n}=$ número de classes de habitat.

A relação entre as espécies e as variáveis de micro-habitat foi realizada por meio de histogramas de frequência, os quais relacionaram cada espécie coletada com as variáveis profundidade, velocidade e substrato, visando interpretar as diferenças nos usos do habitat referentes às espécies dominantes no rio Formoso. Para as variáveis profundidade, velocidade e substrato, os histogramas de frequência relacionaramse a média dos valores extremos de cada classe e o tipo de substrato com o índice padronizado de uso de habitat, que segundo Jowett (2002) deve variar de zero (habitat mínimo) até um (habitat máximo).

Com relação à variável profundidade $(\mathrm{m})$ utilizaramse as seguintes classes: 0,00 a 0,$25 ; 0,25$ a 0,$50 ; 0,50$ a 0,$75 ; 0,75$ a 1,$00 ; 1,00$ a 1,$25 ; 1,25$ a 1,$50 ; 1,50$ a 1,$75 ; 1,75$ a 2,00. E com relação à variável velocidade $\left(\mathrm{m} \mathrm{s}^{-1}\right)$, as seguintes classes foram utilizadas: 0,0 a 0,2 ; 0,2 a 0,$4 ; 0,4$ a 0,$6 ; 0,6$ a 0,$8 ; 0,8$ a 1,$0 ; 1,0$ a 1,$2 ; 1,2$ a 1,4 .

De acordo com Jowett (2002), essa relação permite gerar um Índice de Uso de Habitat (IUH), o qual foi calculado por meio da relaçáo padronizada entre a frequência de uso e a frequência de disponibilidade de habitat, sendo os histogramas suavizados por meio da Curva de Frequência de Kernel (COSTA et al.,
2013). Os resultados dessas curvas são utilizados como dados de entrada em modelos de avaliação de habitat e determinação de vazôes ecológicas (GUEDES et al., 2014; CASTRO et al., 2016). Todas as curvas de uso foram geradas por meio do software HABPRF (Analysis of Habitat Suitability - version 1.0) (JOWETT, 2011).

\section{RESULTADOS E DISCUSSÃO}

As análises das 24 amostras indicaram que as cinco espécies dominantes Astyanax bimaculatus Linnaeus, 1758, Geophagus brasiliensis Quoy \& Gaimard, 1824, Hypostomus affinis Steindachner, 1877, Leporinus steindachneri Eigenmann, 1907 e Trachelyopterus striatulus Steindachner, 1877, contribuíram com mais de $70 \%$ do total de peixes coletadas no rio Formoso.

A distribuição e a abundância das cinco espécies nativas observadas no rio Formoso foram qualitativamente diferentes em relação aos trechos monitorados (Tabela 2), sendo o número de peixes maior no primeiro trecho, onde o regime de escoamento predominante foi supercrítico (ou turbulento) e o ecossistema mais preservado, correspondendo a $58,1 \%$ do número total de indivíduos amostrados.

Observa-se na Tabela 2 que foram coletados mais indivíduos no trecho 1 (58,1\%), comparado ao trecho 2 (41,9\%). As espécies G. brasiliensis (Perciformes, Cichlidae), H. affinis (Siluriformes, Loricariidae) e $L$. steindachneri (Characiformes, Anostomidae) foram dominantes no trecho 1 , e as espécies $A$. bimaculatus (Characiformes, Characidae) e T. striatulus (Siluriformes, Auchenipteridae) foram dominantes no trecho 2. Visualmente o trecho 1 possui uma condição ecossistêmica menos alterada, com margens protegidas por mata ciliar, sem influência direta da urbanização. Já o trecho 2 encontra-se bastante degradado, por meio da influência direta da urbanização, com lançamento de esgoto urbano, uso do solo substituído por pastagens e supressão da vegetação ripária. De acordo com Ferreira e Cassati (2006), dentre os fatores que afetam a qualidade de ambientes lóticos para a ictiofauna destaca-se a perda e a transformação do habitat, que geralmente está associada à supressão da vegetação ripária, com consequente aumento da 
Tabela 2. Número de indivíduos coletados em função das cinco espécies nativas em dois trechos do rio Formoso e seus percentuais de ocorrência (em parênteses)

Espécies dominantes Trecho de rio

\begin{tabular}{llll}
\hline Nome científico & Nome popular & 1 & 2 \\
\hline Astyanax bimaculatus & Lambari-de-rabo-amarelo & $12(15,2)$ & $25(43,9)$ \\
Geophagus brasiliensis & Cará, acará & $18(22,8)$ & $3(5,3)$ \\
Hypostomus affinis & Cascudo & $25(31,6)$ & $11(19,3)$ \\
Leporinus steindachneri & Piau & $20(25,3)$ & $1(1,7)$ \\
Trachelyopterus striatulus & Cumbaca & $4(5,1)$ & $17(29,8)$ \\
\hline Total & & $79(58,1)$ & $57(41,9)$
\end{tabular}

incidência solar e a ausência de determinados itens alimentares, como frutos, sementes e insetos alóctones (MOUNTON et al., 2012).

As espécies de menor porte, $A$. bimaculatus e $T$. striatulus, foram mais presentes no trecho 2 , onde o regime de escoamento é predominantemente fluvial (lento) e o ecossistema degradado. Já as espécies de médio a grande porte, G. brasiliensis, $H$. affinis e L. steindachneri, foram mais presentes no trecho 1, onde o regime de escoamento foi caracterizado como torrencial (rápido) e o ecossistema preservado. Segundo Leal et al. (2013), os indivíduos tendem a ocupar os habitats aos quais estão mais adaptados, sendo geralmente intensa a relação entre as espécies e determinadas variáveis ambientais, como velocidade da água, profundidade e tipo de substrato.

As espécies de peixes de água doce estão presentes em habitats diversos e utilizam as condições abióticas, características hidráulicas, temperatura da água e concentração de oxigênio, como fatores limitantes de distribuição (DAGA et al., 2012). Particularmente no Brasil, a perda de habitat em longo prazo é devido à associação de barramentos dos rios, desmatamento, poluição das águas, práticas agrícolas inadequadas, apresentando como consequência direta a diminuição na disponibilidade de peixes para as comunidades que necessitam desses recursos para sobreviverem e para a riqueza dos ecossistemas (BARLETTA et al., 2010).

A profundidade monitorada nos dois trechos do rio Formoso variou de 0,2 a 2,0 m, sendo a maior frequência de ocorrência dos exemplares entre 0,6 a $1,0 \mathrm{~m}$, correspondendo a $59 \%$ do total de peixes observados (Figura 2). A profundidade na qual as espécies são encontradas é dependente de vários fatores como, por exemplo, as características biológicas, a
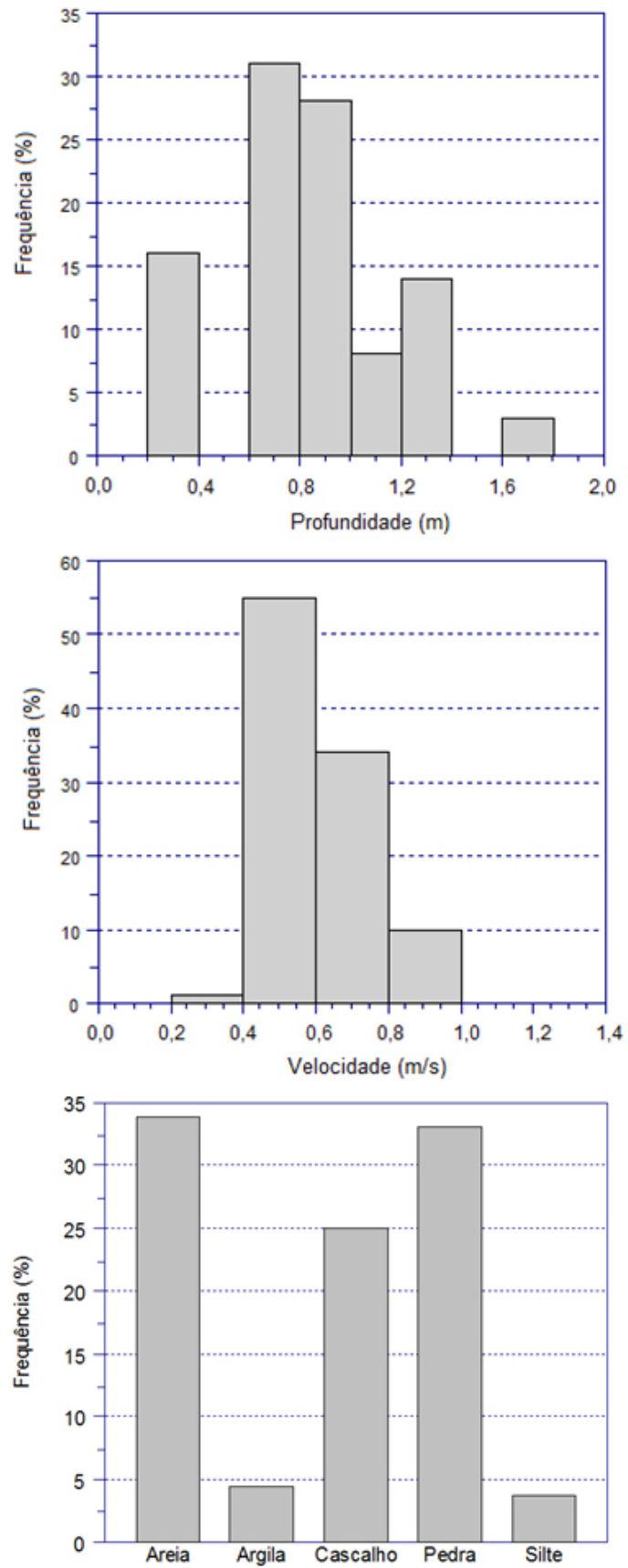

Figura 2. Frequência de ocorrência dos indivíduos (\%) em função das variáveis profundidade $(\mathrm{m})$, velocidade $(\mathrm{m}$ $\mathrm{s}^{-1}$ ) e substrato no rio Formoso 
fase de desenvolvimento (juvenil e adulto), a relação predatória e a disponibilidade de oxigênio. As espécies de menor porte como a $A$. bimaculatus e a T. striatulus são encontradas com maior êxito em profundidades maiores que $0,80 \mathrm{~m}$, dada a sua maior capacidade de permanecer em regimes de escoamento mais lento. Adrian et al. (2001) estudaram a espécie $A$. bimaculatus na área de influência do reservatório de Corumbá (GO) e verificaram que essa espécie possui grande resiliência no curso d'água, principalmente em maiores profundidades do escoamento, sendo capaz de resistir aos impactos causados por obras hidráulicas e escassez de alimentos. De acordo com Hahn et al. (1997) há uma dificuldade de se encontrar indivíduos dessa espécie a grandes profundidades, uma vez que o hábito de se explorar o fundo dos cursos d'água não é comum para o gênero Astyanax.

A velocidade nas seçóes de monitoramento do rio Formoso variou de 0,2 a $1,4 \mathrm{~m} \mathrm{~s}^{-1}$, sendo a maior frequência de ocorrência dos exemplares (55\%) entre 0,4 a $0,6 \mathrm{~m} \mathrm{~s}^{-1}$. Velocidades mais elevadas (> 1,0 $\left.\mathrm{m} \mathrm{s}^{-1}\right)$ náo apresentaram exemplares e velocidades menos elevadas $\left(<0,4 \mathrm{~m} \mathrm{~s}^{-1}\right)$ apresentaram apenas $1 \%$ de exemplares. Da mesma forma que a variável profundidade, a preferência das espécies por certas faixas de velocidade depende, também, das características biológicas, da dinâmica do escoamento, da fase de desenvolvimento e da concentração de oxigênio dissolvido na massa líquida.

Vlach et al. (2005) encontraram relaçáo direta entre o aumento da profundidade e velocidade do escoamento com o aumento da diversidade e quantidade de peixes. Entretanto, pesquisas mostram que somente o estudo da velocidade e da profundidade não é significativo para indicar modificaçôes nas comunidades aquáticas (KAUFMANN; PINHEIRO, 2009), sendo necessária a incorporação de dados de substrato para melhor avaliar essas alterações (HUMPL; PIVNICKA, 2006; COSTA et al., 2013).

A maior frequência de ocorrência de indivíduos encontrada foi para o tipo de substrato areia, com 34\% de ocorrência, seguido dos substratos pedra (33\%) e cascalho (25\%). Os substratos argila e silte foram os que apresentaram menor frequência de exemplares, ambos com $4 \%$. A escassez de pedras e outros subtratos consolidados no trecho 2 no rio Formoso podem indicar maiores restriçóes ambientais que, provavelmente, limitam as assembleias de peixes de médio a grande porte como as espécies $G$. brasiliensis, $H$. affinis e $L$. steindachneri (DUARTE; ARAÚJO, 2000). Garavello e Garavello (2004) explicam que a permanência do gênero Hypostomus no curso d'água está associada à disponibilidade de alimentos, se fixando no fundo do rio para se alimentar de algas presas no subtrato rochoso. Durante o dia, os indivíduos permanecem sob as rochas ou trocos submersos (WEBER, 2003).

Analisando a ocorrência de cada espécie dominante, em função das variáveis profundidade, velocidade e substrato, observa-se na Figura 3 que os exemplares das espécies $A$. bimaculatus e $L$. steindachneri foram capturados com mais sucesso (IUH igual a 1,0 ) na faixa de profundidade do escoamento entre 0,8 e 1,0 $\mathrm{m}$. Entretanto, indivíduos da espécie L. steindachneri também foram capturados (IUH igual a 0,9) em profundidades menores $(0,2$ a $0,4 \mathrm{~m})$. Isso se justifica em função das características biológicas para cada fase de desenvolvimento da espécie. Os indivíduos adultos, por exemplo, necessitam de grandes profundidades para obterem a sua dieta alimentar. Já os indivíduos juvenis necessitam de menores profundidades. Para as espécies $G$. brasiliensis e $H$. affinis, a captura obteve mais sucesso em profundidades entre 0,6 e $0,8 \mathrm{~m}$. A maioria dos exemplares da espécie $T$. striatulus foram encontrados em profundidades do escoamento maiores (1,2 a $1,4 \mathrm{~m})$.

Ao longo das quatro coletas de dados no rio Formoso, os indivíduos das espécies $G$. brasiliensis, $H$. affinis, L. steindachneri e T. striatulus foram capturados com mais sucesso (IUH igual a 1,0) quando a faixa de velocidade da água encontrada variou entre 0,4 a 0,6 $\mathrm{m} \mathrm{s}^{-1}$ (Figura 4).

A exceção, entre as espécies dominantes consideradas nesse estudo, foi os indivíduos da espécie A. bimaculatus com faixa de velocidade entre 0,6 a 0,8 $\mathrm{m} \mathrm{s}^{-1}$. A espécie $G$. brasiliensis foi a única a apresentar exemplares coletados em baixas velocidades $(<0,4 \mathrm{~m}$ $\left.\mathrm{s}^{-1}\right)$ e as espécies $A$. bimaculatus, $H$. affinis e T. striatulus foram as que apresentaram exemplares coletados em velocidades mais altas $\left(>0,8 \mathrm{~m} \mathrm{~s}^{-1}\right)$.

Os exemplares da espécie L. steindachneri foram 

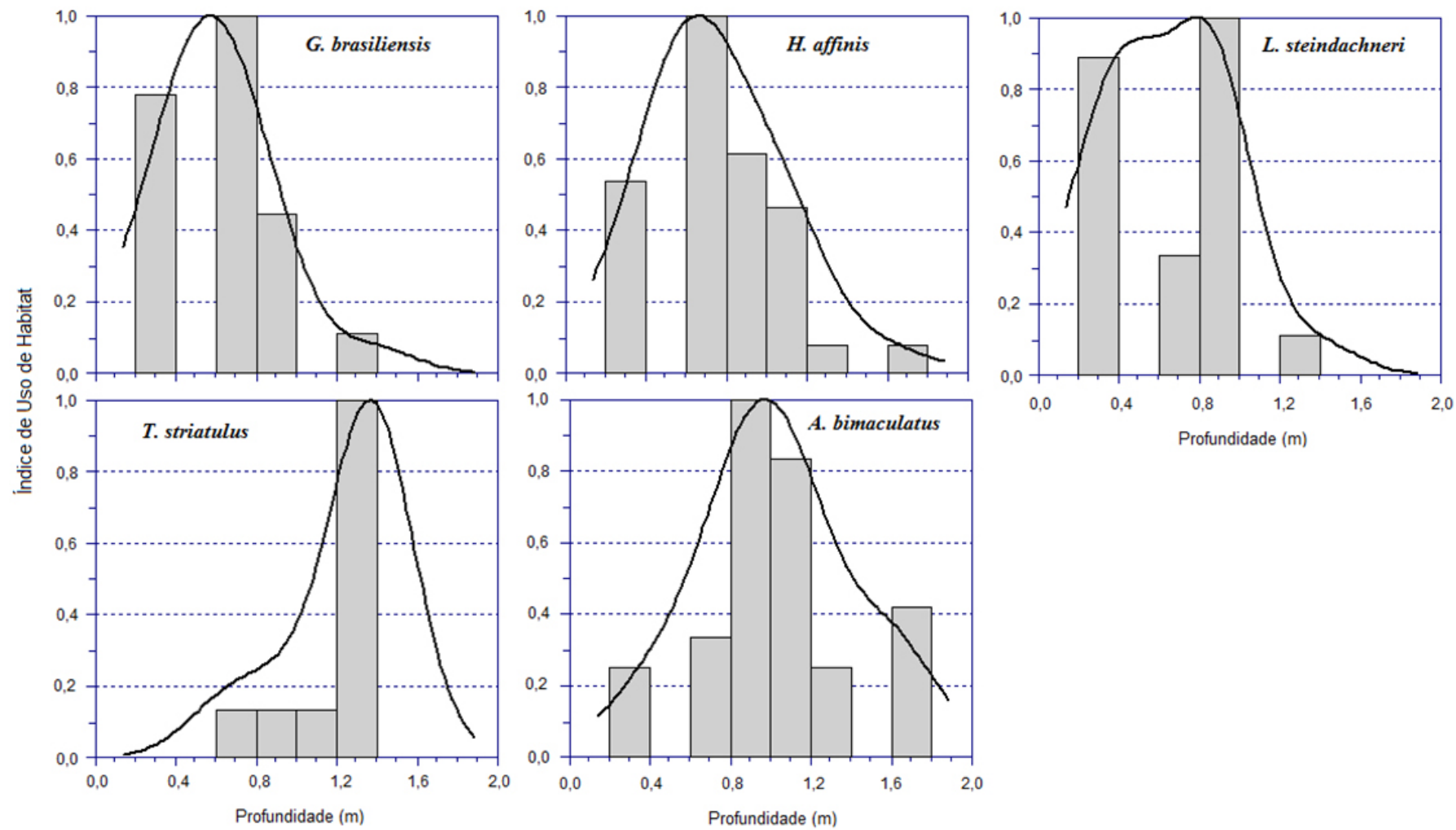

Figura 3. Índice de uso de habitat das espécies de peixes nativos referente à variável profundidade (m) no rio Formoso
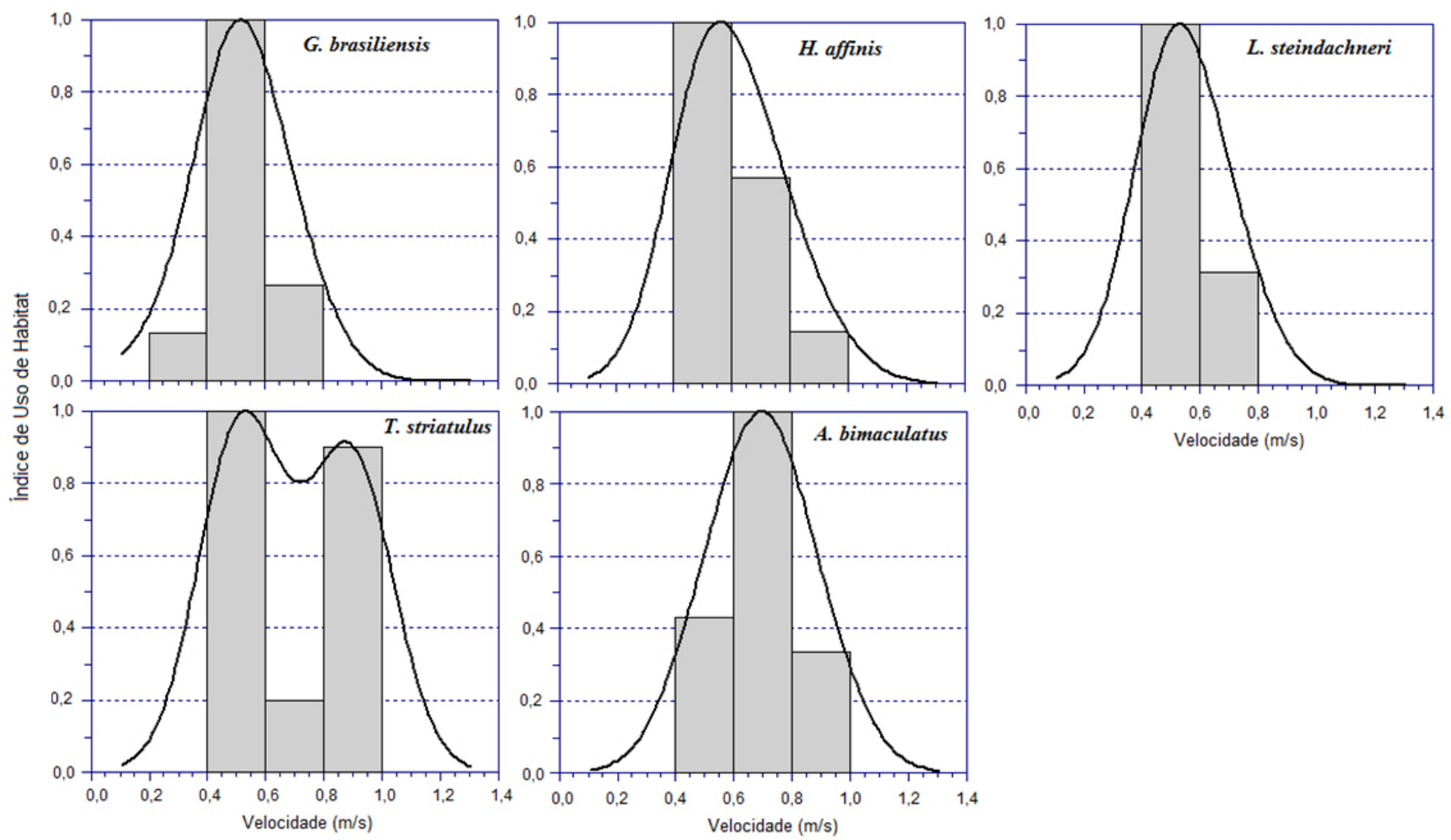

Figura 4. Índice de uso de habitat das espécies de peixes nativos referente à variável velocidade $\left(\mathrm{m} \mathrm{s}^{-1}\right)$ no rio Formoso 

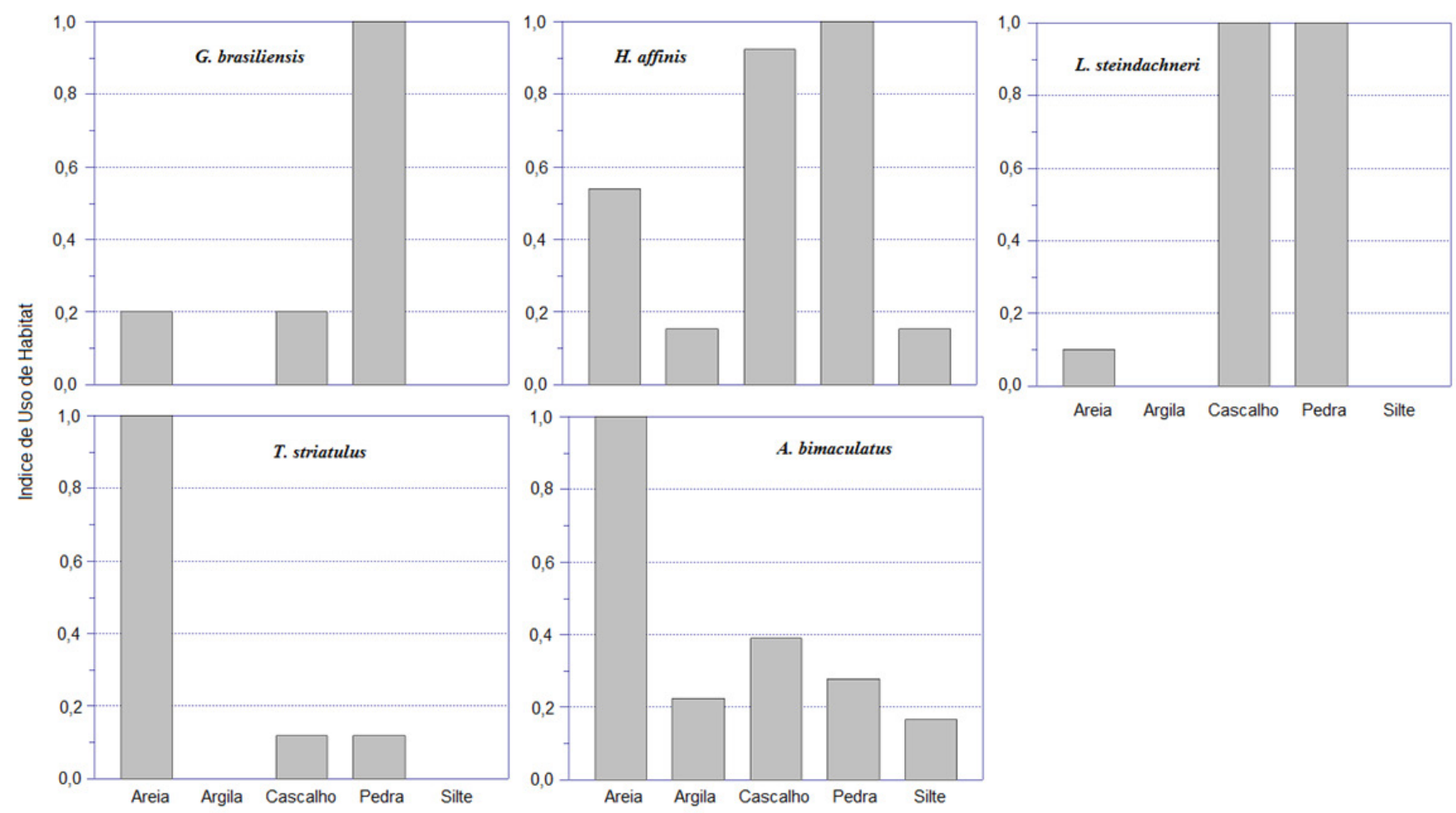

Figura 5. Índice de uso de habitat das espécies de peixes nativos referente à variável substrato no rio Formoso

capturados, quase em sua totalidade, nos substratos classificados como cascalho e pedra (Figura 5). Os indivíduos que se sobressaíram no substrato "pedra" foram os das espécies $G$. brasiliensis e $H$. affinis. Já as espécies $A$. bimaculatus e T. striatulus apresentaram exemplares capturados com mais sucesso no substrato "areia".

Os resultados apresentados mostram que a distribuição dos exemplares coletados no rio Formoso difere em função do local de amostragem e das variáveis velocidade, profundidade e substrato, corroborando a hipótese feita no início do estudo. Por conseguinte, observa-se que o uso por habitats é diferente para cada espécie, o que, segundo Frankham et al. (2012), regula a permanência ou a busca por melhores locais de sobrevivência.

De acordo com Li et al. (2013), as variaçóes de micro-habitat impóem uma importante interface entre o ecossistema aquático e o terrestre, sendo passível de exploração direta pelas espécies piscícolas. A relação água-solo tem sido apontada com uma região importante para a ictiofauna devido a sua quantidade de micro-habitats como, pedras, troncos, galhos, folhas e depósitos de sedimentos que servem como locais de abrigo para os peixes.
Os Índices de Uso de Habitat (IUH) obtidos neste estudo são imprescindíveis nos estudos de modelagem ecohidrológica, uma vez que são dados de entrada nos modelos, permitindo relacionar as interferências hidrológicas no ecossistema aquático. Os índices gerados permitem analisar cenários individuais por espécies ou análises conjuntas. Guedes et al. (2014) determinaram o regime de vazóes ecológicas no rio Formoso, MG, a partir dos IUH das espécies Hypostomus aurogutattus, Leporinus mormyrops e Oligosarcus hepsetus. Castro et al. (2016) estimaram o regime de vazóes ecológicas na bacia do rio de Ondas, $\mathrm{BA}$, utilizando o IUH de diferentes espécies do gênero Hypostomus.

A quantificação das relações entre vazão e respostas ecológicas é a etapa mais importante dos estudos ecohidrológicos (WANG et al., 2013). A determinação do regime de vazóes ecológicas deve apresentar uma base ecológica, sendo estudada uma ou mais espécies, sendo possível analisar como as variaçóes de vazão interferem na dinâmica do ecossistema aquático. Embora bastante discutido na comunidade científica ainda se encontram estudos exclusivamente com dados hidrológicos e interpretados como vazóes ecológicas (GUEDES et al., 2014). Posteriormente, os 
resultados dos estudos de modelagem ecohidrológica permitem subsidiar análises de outorga de uso dos recursos hídricos. Assim, os IUHs são fundamentais para garantir esses estudos futuros.

Apesar de os resultados obtidos neste estudo serem específicos para o rio Formoso, os padróes de uso de habitat pelas espécies dominantes consideradas podem ser consistentes e transferíveis para outras bacias hidrográficas neotropicais. Os usos de habitat descritos neste trabalho apresentam um avanço no conhecimento ao associar cada espécie com um determinado uso de habitat, e estabelecem uma perspectiva promissora para os estudos que visam compatibilizar os diversos usos dos recursos hídricos em regióes neotropicais.

\section{CONCLUSÕES}

As espécies de peixes monitoradas no presente estudo apresentaram exemplares em diferentes condiçóes de preservação de mananciais e regime de escoamento hidráulico. Entretanto, as espécies de menor porte, $A$. bimaculatus e $T$. striatulus, apresentaram mais indivíduos no trecho mais degradado e escoamento fluvial, e as espécies de maior porte, G. brasiliensis, $H$. affinis e L. steindachneri, apresentaram mais indivíduos no trecho mais preservado e escoamento torrencial. Isso é uma indicação da preferência dessas espécies pelas características analisadas nesse estudo. Entretanto, estudos mais específicos, com a análise de outras variáveis, como a concentração de oxigênio dissolvido na massa líquida, característica da dieta alimentar de cada espécie, relação predatória entre espécies, devem ser analisados visando aumentar a certeza nos resultados obtidos e a reprodutibilidade para outras bacias hidrográficas. Somente com esses estudos adicionais é possível explicar as variaçóes encontradas de profundidade, velocidade e substrato por cada uma das espécies analisadas. Os Índices de Uso de Habitat e as curvas de frequência obtidos neste estudo poderão ser utilizados futuramente para estimar o regime de vazões ecológicas no rio Formoso, considerando a variabilidade de micro-habitat das espécies analisadas, e subsidiar estudos de outorga de uso dos recursos hídricos, além de promover a preservação ecológica dos mananciais. Dessa forma, os índices e as curvas são uma ferramenta imprescindível na tomada de decisão de órgãos ambientais.

\section{AGRADECIMENTOS}

Os autores agradecem o apoio financeiro concedido pelo Conselho Nacional de Pesquisa (CNPq), pela Coordenação de Aperfeiçoamento de Pessoal de Nível Superior (CAPES) e pela Fundação de Amparo à Pesquisa do Estado de Minas Gerais (FAPEMIG), que viabilizaram a realização deste trabalho, além do Instituto Estadual de Florestas (IEF), que concedeu a licença de pesca científica ( $\mathrm{N}^{\circ}$ 222/11), e aos estudantes Udson Santos, Nicholas Walker, Priscilla Caroline Silva, Frederico Fernandes Ferreira e Natália Travenzoli, que participaram das atividades de campo.

\section{LITERATURA CITADA}

ANDRIAN, I. F.; SILVA, H. B. R.; PERETTI, D. Dieta de Astyanax bimaculatus (Linnaeus, 1758) (Characiformes, Characidae), da área de influência do reservatório de Corumbá, Estado de Goiás, Brasil. Maringá, v.23, p.435-440, 2001.

BARLETTA, M.; JAUREGUIZAR, A. J.; BAIGUN, C.; FONTOURA, N. F.; AGOSTINHO, A. A.; ALMEIDA-VAL, V. M. F.; VAL, A. L.; TORRES, R. A.; JIMENES-SEGURA, L. F.; GIARRIZO, T.; FABRÉ, N. N.; BATISTA, V. S.; LASSO, C.; TAPHORN, D. C.; COSTA, M. F.; CHAVES, P. T.; VIEIRA, J. P.; CORRÊA, M. F. M. Fish and aquatic habitat conservation in South America: a continental overview with emphasis on neotropical systems. Journal of Fish Biology, v.76, p.21182176, 2010.

BARROCA, T. M. ; ARANTES, F. P.; MAGALHÃES, B. F.; SIQUEIRA, F. F.; HORTA, C. C. R.; PENA, I. F.; DERGAM, J. A.; Kalapothakis, E. Genetic diversity and population structure of Prochilodus costatus and Prochilodus argenteus preceding dam construction in the Paraopeba River, São Francisco River Basin, Minas Gerais, Brazil. Open Journal of Genetics, v.2, p.121-130, 2012.

BOVEE, K. D. A Guide to Stream Habitat Analysis Using the Instream Flow Incremental Methodology. Instream Flow Information Paper No. 12. U.S. Fish and Wildlife Service. FWS/ OBS-82/26, 1982.

BRITSKI, H. A.; SATO, Y.; ROSA, A. B. S. Manual de Identificaçáo de Peixes da Regiáo de Três Marias (com publicaçáo chaves de identificaçáo para os peixes da bacia do Sáo Francisco). Brasília: Câmara dos Deputados, CODEVASF, Divisão de Piscicultura e Pesca, 1986. 143 p.

CASTRO, E. R. R. S., MOREIRA, M. C.; SILVA, D. D. Environmental flow in the River Ondas basin in Bahia, Brazilian Cerrado. Environmental Monitoring Assessment, v.188, p.68-77. 2016.

COSTA, M. R. da; MATTOS, T. M.; BORGES, J. L.; ARAÚJO, F. G. Habitat preferences of common native fishes in a tropical river in Southeastern Brazil. Neotropical Ichthyology, v.11, p.871-880, 2013. 
DAGA, S. V., GUBIANI, É. A., CUNICO, A. M.; BAUMGARTNER, G. Effects of abiotic variables on the distribution of fish assemblages in streams with different anthropogenic activities in southern Brazil. Neotropical Ichthyology, v.10, p.643-652, 2012.

DEVINE, B. M.; MUNDAY, P. L. Habitat preferences of coral-associated fishes are altered by short-term exposure to elevated CO. Marine Biology, v.160, p.1955-1962, 2013.

DUARTE, S.; F. G. ARAÚJO. Distribuiçáo espacial e temporal de Hypostomus affinis na Represa de Ribeiráo das Lajes. Acta Biologica Leopoldensia, v.22, p.261-276, 2000.

FERREIRA, C. de P.; CASATTI, L. Influência da estrutura do hábitat sobre a ictiofauna de um riacho em uma microbacia de pastagem, Sáo Paulo, Brasil. Revista Brasileira de Zoologia, v.23, p.642-651, 2006.

FRANKHAM, R; BALLOU, J D; DUDASH, M R; ELDRIDGE, M D B; FENSTER, C B; LACY, R C; MENDELSON III, J R; PORTON, I J; RALLS, K; RYDER, O A. Implications of different species concepts for conserving biodiversity. Biological Conservation, v. 153, p.25-31, 2012.

GARAVELLO, J. C.; GARAVELLO, J. P. Spatial distribution and interaction of four species of the catfish genus Hypostomus Lacepède with bottom of Rio Sáo Francisco, Canindé do São Francisco, Sergipe, Brazil (Pisces, Loricariidae, Hypostominae). Brazilian Journal of Biology, v.64, p.591-598, 2004.

GUEDES, H. A. S.; SILVA, D. D.; DERGAM, J. A.; ELESBON, A. A. A. Estabelecimento do regime de vazóes ecológicas no rio Formoso/MG com base em espécies neotropicais. Revista Brasileira de Recursos Hídricos, v. 19, p.72-82, 2014.

HAHN, N. S.; ANDRIAN, I. F.; FUGI, R.; ALMEIDA, V. L. Ecologia trófica. In: VAZZOLER, A. E. A. de M.; AGOSTINHO, A. A.; HAHN, N. S. A planície de Inundaçáo do alto rio Paraná: aspectos físicos, biológicos e socioeconômicos. Maringá: EDUEM. 1997. p.209-228.

HUMPL, M.; PIVNICKA, K. Fish assemblages as influenced by environmental factors in streams in protected areas of the Czech Republic. Ecology of Freshwater Fish, v.15, p.96-103, 2006.

JOWETT, I. G. In-stream habitat suitability criteria for feeding inanga (Galaxias maculatus). Journal of Marine and Freshwater Research, v.36, p.399-407, 2002.

JOWETT, I. G. Computer software - HABPFR - version 1.0. New Zealand Foundation for Science Research and Technology and Regional Councils, New Zealand. Disponível em: $<$ http:// www. jowettconsulting.co.nz/home/habprf/>. Acesso em: 01.04.2015. 2011.

KAUFMANN, V.; PINHEIRO, A. Relaçóes entre diversidade íctia e fatores hidrodinâmicos de um riacho na bacia do rio Uruguai. Biota Neotropica, v.9, p.47-53, 2009.

LEAL, C. G.; JUNQUEIRA, N. T.; SANTOS, H. de A. e;
POMPEU, P. S. Variaçóes ecomorfológicas e de uso de habitat em Piabina argentea (Characiformes, Characidae) da bacia do rio das Velhas, Minas Gerais, Brasil. Ilheringia, Série Zoologia, v.103, p.222-231, 2013.

MARSILI-LIBELLI, S.; GIUSTI, E.; NOCITA, A. A new instream flow assessment method based on fuzzy habitat suitability and large scale river modeling. Environmental Modelling \& Software, v.41, p.27-38, 2013.

MENEZES, N. A. Três espécies novas de Oligosarcus Günther, 1864 e redefiniçáo taxonômica das demais espécies do gênero (Osteichthyes, Teleostei, Characidae). Boletim de Zoologia, v.11, p.1-39, 1987.

MOUNTON, A. M.; BUYSSE, D.; STEVENS, M.; VAN DEN NEUCKER, T.; COECK, J. Evaluation of Riparian Habitat Restoration in a Lowland River. River Research and Applications, v.28, p.845-857, 2012.

REIS, R. E.; KULLANDER, S. O.; FERRARIS JR., C. F. (Org.). Check list of the freshwater fishes of south and central America. Porto Alegre: EDIPUCRS, 2003. 742 p.

SOUZA, A. T.; DIAS, E.; NOGUEIRA, A.; CAMPOS, J.; MARQUES, J. C.; MARTINS, I. Population ecology and habitat preferences of juvenile flounder Platichthys flesus (Actinopterygii: Pleuronectidae) in a temperate estuary. Journal of Sea Research, v.79, p.60-69, 2013.

STALNAKER, C.; LAMB, B. L.; HENRIKSEN, J.; BOVEE, K.; BARTHLOW, J. The Instream Flow Incremental Methodology. A Primer for IFIM. U.S. Departament of Interior. National Biological Service, Washington, D.C, 1995.

TERESA, F. B.; CASATTI, L. Development of habitat suitability criteria for Neotropical stream fishes and an assessment of their transferability to streams with different conservation status. Neotropical Ichthyology, v.11, p.395-402, 2013.

TERRA, B. F., SANTOS, A. B. I.; ARAÚJO, F. G. Fish assemblage in a dammed tropical river: an analysis along the longitudinal and temporal gradients from river to reservoir. Neotropical Ichthyology, v.8, p.599-606, 2010.

VLACH, P.; DUŠEK, J.; ŠVÁTORA, M.; MORAVEC, P. Fish assemblage structure, habitat and microhabitat preference of five fish species in a small stream. Folia Zoologica, v.54, p.421431, 2005.

WANG, J; DONG, Z; LIAO, W; LI, C; FENG, S; LUO, H; PENG, $Q$. An environmental flow assessment method based on the relationships between flow and ecological response: $A$ case study of the Three Gorges Reservoir and its downstream reach. Science China, v.56, p.1471-1484, 2013.

WEBER, C. Subfamily Hypostominae. In: REIS, R. E.; KULLANDER, S. O.; FERRARIS-JR, C. J. Check list of the freshwater fishes of South and Central America. Porto Alegre, Editora da Pontifícia Universidade Católica do Rio Grande do Sul. 2003. p. 351-372. 